\title{
Protocatechuic acid decreased telomerase reverse transcriptase (TERT) expression in DMBA-induced liver carcinogenesis mice model
}

\author{
Samar El-Shaheed ${ }^{1)}$, Heba Sahyon ${ }^{2)}$, Magdy Youssef ${ }^{1)}$ and Amr Negm ${ }^{1,3)}$ \\ 1) Biochemistry division, Chemistry Department, Faculty of Science, Mansoura University, Egypt \\ ${ }^{2)}$ Biochemistry division, Chemistry Department, Faculty of Science, Kafr Elsheikh University, Egypt \\ ${ }^{3)}$ Department of Chemistry, College of Science, King Faisal University, Al-Ahasa, Saudi Arabia
}

\begin{abstract}
Background: The protocatechuic acid (PCA) is a natural polyphenolic antioxidant commonly distributed in plants and is considered as one of the main ingredients of some traditional herbal medicine. PCA has many pharmacological effects that may be closely linked to its antioxidant activity. Herein, we newly conducted this animal experiment to evaluate the therapeutic potentials of PCA on the 7,12-Dimethylbenzanthracene (DMBA) -induced hepatocarcinogenesis in the mouse model. Additionally, the in vitro antioxidant activity of PCA was estimated.

Material and methods: To induce the hepatocarcinogenesis, DMBA (35 mg/kg, once per week) was orally administered for 4 weeks in the tumor test groups. After six weeks, the administration of PCA (100 mg/kg/day) was started for 4 weeks. Mice were sacrificed after ten weeks for measuring and comparing the serum hepatic enzymes level, antioxidant markers and telomerase reverse transcriptase (TERT) gene expression between the mice in DMBA with PCA (PCA group) and DMBA without PCA (non-PCA group). Also, the antioxidant activity of PCA was measured in vitro using two different antioxidant assays.

Results: The elevation of all liver enzymes and other hepatic biomarkers observed in DMBA group were significantly suppressed in PCA-treated group. In addition, the TERT gene expression in liver tissue was significantly decreased in PCAtreated group. Our results also showed that the PCA exhibited a high antioxidant ability in vitro comparable to the positive control ascorbic acid.

Conclusion: The protocatechuic acid decreased TERT expression and oxidative stress in DMBA-induced liver carcinogenesis mice model.
\end{abstract}

Keywords: Protocatechuic acid, liver carcinogenesis, antioxidant, telomerase reverse transcriptase.

(Received January 23, 2020; Accepted April 8, 2020)

\section{Introduction}

The liver is the central organ that metabolize many potentially toxic chemicals, where they are concentrated and become activated ${ }^{1}$. Liver cancer is one of the most mortal tumors worldwide. Globally, it is the second leading cause of death in cancer related death cases ${ }^{2)}$.

7,12-Dimethylbenzanthracene, also known as DMBA, is a prominent genotoxic procarcinogen that is activated in the liver by detoxification enzymes to be transformed into an utmost carcinogen ${ }^{3)}$. DMBA exposure can cause several toxicological alterations in the liver, including oxidative damage via reactive oxygen species (ROS) release and formation of carcinogenic metabolites ${ }^{4}$. Various studies have dealt with the toxic effect of DMBA on the liver ${ }^{5-7)}$.

Correspondence to: Samar El-Shaheed, Biochemistry division, Chemistry Department, Faculty of Science, Mansoura University, Egypt. P.B. Box 33516, Tel: +201002427384; Email: samarhosny2014@yahoo.com
Recently, herbal therapies have attracted huge attention due to their wide range of biological and therapeutic properties $^{8}$. The protocatechuic acid (PCA, 3,4-dihydroxybenzoic acid), is a natural polyphenolic antioxidant that is found in fresh fruits, vegetables, and extra virgin olive oil as well as in processed food such as red and white grape wine and in herbal medicine ${ }^{9,10)}$. PCA has been demonstrated to have an effective anticancer activity against various cancer inducing-chemicals in different tissues, such as liver, which thought to be closely related to its antioxidant activity ${ }^{11}$.

Telomerase is a reverse transcriptase enzyme that synthesizes TTAGGG telomeric repeats on the ends of chromosomes to maintain the length of tolemere ${ }^{12)}$. Most cancer cells have telomerase activity which supports their unlimited proliferation ${ }^{13)}$. Telomerase reverse transcriptase (TERT) is a catalytic subunit of telomerase, which, together with the telomerase RNA (TR), comprises the most important unit of the telomerase complex ${ }^{14)}$. It is recognized that the activity of telomerase is strongly 
regulated by the level of TERT synthesis ${ }^{15)}$. In the current study, our attention was directed to evaluate the in vitro antioxidant capacity of PCA. Additionally, we used DMBA-induced liver carcinogenesis mouse model to explore the telomerase inhibition by PCA.

\section{Materials and methods}

\section{Allocation of intervention and control setting}

Thirty-two mice were divided into 4 groups (8 animals each); normal control, PCA, DMBA, and DMBA with PCA group. Mice were given olive oil once/week for 4 weeks and daily normal saline for 4 weeks in normal control group. Mice received PCA at a dose of $100 \mathrm{mg} / \mathrm{kg}$ body weight (b.w.)/day by intraperitoneal injection until the end of the experiment in PCA group. Hepatocarcinogenesis was induced in DMBA group in which DMBA was orally administrated at a dose of 35 $\mathrm{mg} / \mathrm{kg}$ b.w., dissolved in $100 \mu \mathrm{l}$ olive oil, once a week for four weeks as reported by the protocol of Mansour et al. ${ }^{16)}$ with slight modifications. Mice received PCA (dissolved in normal saline) at a dose of $100 \mathrm{mg} / \mathrm{kg}$ b.w./day by intraperitoneal injection after six weeks of DMBA administration (starting of promotion stage) and continued for four weeks in DMBA with PCA group. The dose of PCA was selected based on the acute toxicity study achieved in our laboratory according to the Organization for Economic Co-operation and Development (OECD) (2008) guidelines ${ }^{17)}$ and on the basis of other published report ${ }^{18)}$. The body weights of all mice were estimated at the starting and end of the experiment.

\section{Outcomes}

\section{Biomarkers related to liver function}

Twenty-four hours after the last treatment, mice were sacrificed under ether anesthesia, blood was withdrawn directly by cardiac puncture and the sera were separated by centrifugation at $3000 \mathrm{rpm}$ for $15 \mathrm{~min}$. Serum alanine transaminase (ALT), aspartate transaminase (AST) and $\gamma$-glutamyl transferase $(\gamma \mathrm{GT})$ were assayed by kinetic photometric methods using commercial kits. Total bilirubin (T.B) and albumin (Alb) were assayed by colorimetric methods using commercial kits. Alpha fetoprotein (AFP) level was estimated using ready-made Enzymelinked immunosorbent assay kit (ELISA), according to the manufacturer's protocol. Liver from each mouse was immediately removed after dissection and weighed. The relative liver weight was also calculated.

\section{Biomarkers related to hepatic antioxidant status}

The liver tissues were precisely weighed, homogenized in cold phosphate buffer ( $\mathrm{pH}$ 7.4). After centrifugation for $10 \mathrm{~min}$. at $5,000 \mathrm{rpm}$ at $4^{\circ} \mathrm{C}$, the supernatants (homogenates) were utilized for antioxidant and malondialdehyde (MDA) determination. Tissue non-enzymatic reduced glutathione (GSH) was determined by using 5,5'-dithio 2-p-nitrobenzoic acid (DTNB) method as stated by Beutler et al. ${ }^{19)}$. Superoxide dismutase (SOD) activity in liver homogenates was determined spectrophotometrically using commercial kit according to the method described by Nishikimi et al. ${ }^{20)}$. The activity of catalase (CAT) was determined spectrophotometrically using commercial kit according to the method reported by Aebi $^{21)}$ and Fossati et al. ${ }^{22)}$. Determination of MDA in liver homogenates was carried out as previously described $^{23)}$.

\section{In-vitro free radical scavenging activity}

The in-vitro free radical scavenging activity of PCA was examined by 2,2'-azino-bis (3-ethylbenzothiazoline6-sulfonic acid (ABTS) and 2,2-diphenyl-1-picrylhydrazyl (DPPH) radical scavenging activity using ascorbic acid (Vitamin C) as a standard. The ABTS assay was performed following the reported procedure of Re et al. ${ }^{24)}$. DPPH assay was determined according to the method of Mavie et al. ${ }^{25)}$. The antioxidant capacity of PCA was expressed as percent inhibition of absorbance at 734 and $490 \mathrm{~nm}$, respectively.

\section{TERT gene expression}

TERT gene expression was estimated by quantitative Real Time-Polymerase Chain Reaction (RT-PCR). Total ribonucleic acid (RNA) was isolated from liver tissues using RNeasy Mini Kit (Qiagen Inc., San Diego, USA) following the manufacturer's instructions. The synthesis of complementary-DNA (cDNA) was carried out using high capacity cDNA reverse transcription kit (ThermoFisher Scientific, Waltham, USA), following the manufacturer's instructions. Finally, the quantitative RT-PCR was performed using SYBR Green PCR Master Mix (ThermoFisher Scientific) on StepOnePlus RT-PCR System (ThermoFisher Scientific). The relative amount of RNA was calculated using the 2-Delta Delta CT method ${ }^{26)}$. The gene expression was normalized with Glyceraldehydes-3-phosphate dehydrogenase (GAPDH) and results were expressed as fold change related to normal group. The gene-specific primers used for PCR amplification are listed in Table 1.

\section{Statistical analysis and ethical policy}

The analysis of data was carried out by GraphPad Prism software (Version 6.0). The results are expressed as mean \pm standard error mean (SEM). One-way analysis of variance (ANOVA) was used to determine the significance between different groups followed by Dunnett's test (multiple comparison versus control). A P-value of $<0.05$ was statistically significant. The ethical standard approval was taken by the institutional animal ethics committee of guidelines at Mansoura University, Egypt (cod no. $\mathrm{Ph} / \mathrm{chem}$. 19-00001), which are in accordance with the Guide for the Care and Use of Laboratory Animals published by the National Academy of Sciences. 
Table 1 The primer sequences of the studied genes.

\begin{tabular}{c|c|c|c}
\hline Gene & & Sequence $\left(\mathbf{5}^{\prime}-\mathbf{3}^{\prime}\right)$ & Product length \\
\hline \multirow{2}{*}{ TERT } & $\begin{array}{c}\text { Forward } \\
\text { Reverse }\end{array}$ & $\begin{array}{c}\text { 5'-CGG AAG AGTGTC TGG AGC AA -3' } \\
\text { 5'- GGA TGA AGC GGAGTC TGG A -3' }\end{array}$ & 145 \\
\hline \multirow{2}{*}{ GAPDH } & $\begin{array}{c}\text { Forward } \\
\text { Reverse }\end{array}$ & $\begin{array}{c}\text { 5'- ACCACAGTCCATGCCATCAC -3' } \\
\text { 5'- TCCACCACCCTGTTGCTGTA -3' }\end{array}$ & \multirow{2}{*}{452} \\
\hline
\end{tabular}

Table 2 Changes in body weight $(\mathrm{g})$ and relative liver weight/ $100 \mathrm{~g}$ b.w. in the control and different groups treated with PCA

\begin{tabular}{c|c|c|c}
\hline Groups & Initial body weight & Final body weight & Relative liver weight (g\%) \\
\hline $\begin{array}{c}\text { Normal control } \\
\text { \% }\end{array}$ & $23.38 \pm 1.49$ & $36.92 \pm 2.66$ & $4.28 \pm 0.31$ \\
\hline PCA & $22.99 \pm 2.03$ & $57.9 \%$ & $4.48 \pm 0.29^{\mathrm{a}}$ \\
\% & & $56.45 \pm 2.61$ & \\
\hline DMBA & $26.43 \pm 2.8$ & $29.17 \%$ & $6.38 \pm 0.66^{*}$ \\
$\%$ & $23.66 \pm 1.4$ & $10.8 \%$ & $4.55 \pm 0.31^{\#}$ \\
\hline DMBA + PCA & & $36.83 \pm 3.89$ & \\
\hline
\end{tabular}

Data represents the means \pm SEM in each group.

$\%$ of change from initial body weight.

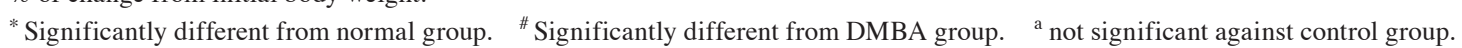

Table 3 Effect of PCA on serum liver function and AFP level in different experimental groups

\begin{tabular}{c|c|c|c|c|c|c}
\hline Groups & ALT (U/L) & AST (U/L) & GGT (U/L) & Alb (g/dl) & T.B(mg/dl) & AFP $(\mathbf{p g} / \mathbf{m l})$ \\
\hline Normal & $35.6 \pm 1.85$ & $105.7 \pm 2.6$ & $4.9 \pm 0.4$ & $3.60 \pm 0.1$ & $0.45 \pm 0.05$ & $34.7 \pm 2.5$ \\
\hline PCA & $38.5 \pm 1.5^{\mathrm{a}}$ & $108.2 \pm 7.06^{\mathrm{a}}$ & $4.5 \pm 1.1^{\mathrm{a}}$ & $3.5 \pm 0.12^{\mathrm{a}}$ & $0.5 \pm 0.04^{\mathrm{a}}$ & $34.2 \pm 1.5^{\mathrm{a}}$ \\
\hline DMBA & $135.2 \pm 6.5^{*}$ & $262.6 \pm 24.8^{*}$ & $33.5 \pm 1.6^{*}$ & $2.6 \pm 0.2^{*}$ & $0.9 \pm 0.3^{*}$ & $109.8 \pm 15.8^{*}$ \\
\hline DMBA + PCA & $47.08 \pm 1^{\#}$ & $136.3 \pm 2.8^{\#}$ & $10.4 \pm 0.4^{\#}$ & $3.32 \pm 0.1^{\#}$ & $0.52 \pm 0.06^{\#}$ & $55.3 \pm 3.6^{\#}$ \\
\% & $-65 \%$ & $-48 \%$ & $-69 \%$ & $30 \%$ & $-43 \%$ & $-50 \%$ \\
\hline
\end{tabular}

Data represents the means \pm SEM in each group.

$\%$ of change from carcinogen group.

* Significantly different from normal group. $\quad$ "Significantly different from DMBA group. $\quad{ }^{\text {a }}$ not significant against control group.

\section{Drugs, measuring kits and housing of animals}

PCA was obtained from, Xi'an Nate Biological Technology Co. (Wuhan, China). ABTS, DPPH, DTNB and TBA were obtained from Sigma-Aldrich Inc. (Saint Louis, USA). ALT and AST diagnostic kits were obtained from HUMAN Company (Cairo, Egypt). $\gamma$ GT was assayed by kinetic photometric method using kit supplied by MyBiosource, Inc. (San Diego, USA). SOD and CAT were purchased from Biodiagnostic Co. (Cairo, Egypt). AFP ELISA kit was purchased from CUSABIO Inc. (Wuhan, China).

Thirty-two adult male swiss albino mice (Mus musculus) weighing from 22 to $26 \mathrm{~g}$, were used and housed in plastic cages. They were kept in 12/12 h light/dark cycle at $22 \pm 2^{\circ} \mathrm{C}$ with free access to water and standard CHOW diet ad libitum. They were kept under observation for one week prior to starting the experiment.

\section{Results}

\section{Clinical findings}

The initial and final body weights and the relative liver weights (g liver/100 g b.w.) of the different groups are listed in Table 2. A slight elevation in final body weight was noticed in DMBA-administrated group with
$10.8 \%$ change in comparison to the initial weight. The treatment of the DMBA group with PCA increased the final body weight with $55.7 \%$ change in comparison to the initial weight. The relative liver weight in DMBA group was markedly increased $(p<0.001)$ to record 6.38 \pm 0.66 when compared to normal control group. At the meantime, the treatment of DMBA group with PCA significantly reduced $(p<0.001)$ the relative liver weight to record $4.55 \pm 0.31$ in comparison with DMBA administrated group but it was not significantly affected in the PCA normal treated group.

\section{Acute toxicity}

It was observed that PCA was safe up to a dose level of $2000 \mathrm{mg} / \mathrm{kg}$. No Mortality and toxicity were induced at this level in all mice during 14-day observation period. One-tenth of the maximum dose (100 mg/ kg b.w.) of the PCA was selected for evaluation of anticancer potential.

\section{Liver function}

Results in Table 3 showed that the DMBA group exhibited a significant increase $(p<0.001)$ in the activities of ALT, AST, $\gamma \mathrm{GT}$, and T. B level with a decrease in albumin level in comparison with that of normal group. The treatment with PCA after DMBA administration 


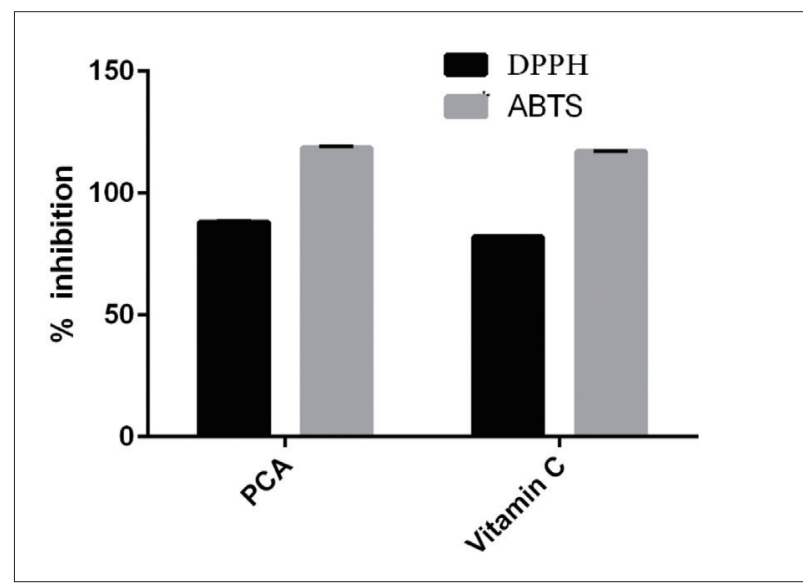

Fig. 1 DPPH and ABTS radical scavenging activity of PCA

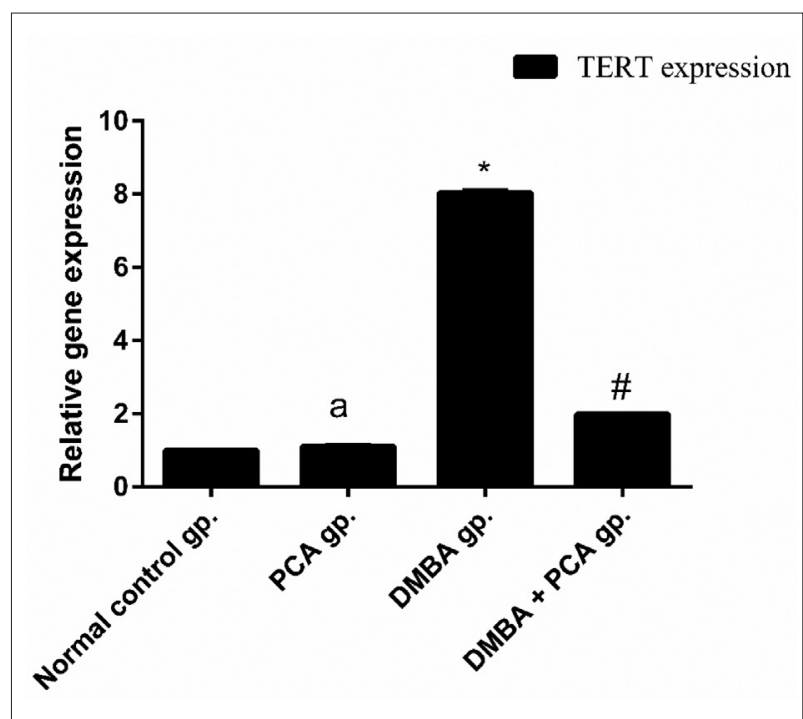

Fig. 3 The TERT expression on the liver

The relative gene expression of normal control group was defined as 1. Data represents the means \pm SEM in each group. * Significantly different from control group. "Significantly different from DMBA group. ${ }^{a}$ not significant against control group.

exhibited a significant decline in serum activities of ALT, AST, $\gamma \mathrm{GT}$, and T. B level, with an increase in albumin level in comparison to DMBA group, but it was not significantly affected in the PCA normal treated group.

Serum level of AFP in control and treated groups was also displayed in Table 3. A significant elevation in AFP level was clearly observed in DMBA-administrated group compared to control normal group $(p<0.001)$. Meanwhile, there was a significant depletion in AFP level after administration of PCA in the DMBA group when compared to model group. Administration of PCA to normal mice resulted in a non-significant change in

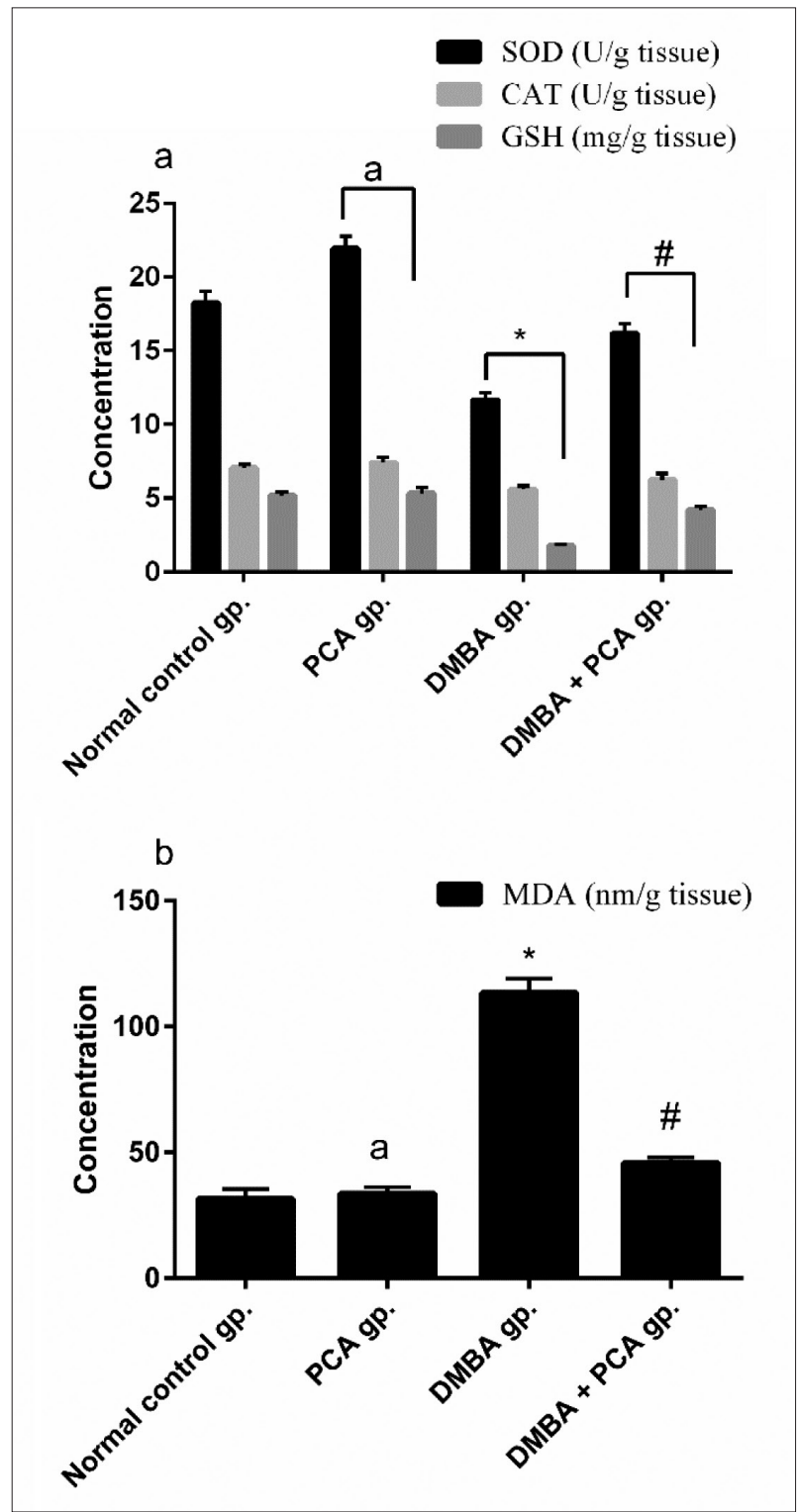

Fig. 2 Antioxidant marker and MDA on the liver (a) antioxidant markers, (b) MDA in normal and DMBA-induced liver cancer in mice. Data represents the means \pm SEM in each group. * Significantly different from control group. "Significantly different from DMBA group. ${ }^{a}$ not significant against control group.

serum AFP level in comparison to normal group. In-vitro free radical scavenging activity and hepatic antioxidant status

The in vitro DPPH and ABTS-scavenging capacities of PCA were 88.1 and $118.45 \%$ at the tested concentration $(1 \mathrm{mg} / \mathrm{ml})$, respectively. Furthermore, the ascorbic acid (Vitamin $\mathrm{C}$ ) equivalent antioxidant capacities of the PCA were 73.7 and $117 \%$ in DPPH and ABTS assays, respectively (Fig. 1). The hepatic antioxidant status in animal experiments was displayed in Fig. 2. A significant reduction in the activities of SOD and CAT along with GSH depletion was clearly noticed in DMBA- 
administrated group in comparison to control healthy group $(p<0.001)$. At the same time, a prominent elevation of the MDA level $(p<0.001)$ was also noticed in the same group in comparison to normal treated group. Conversely, the treatment with PCA after DMBA administration significantly increased the antioxidant enzymes activities and cellular content of GSH with a significant decrease in MDA level when compared to carcinogen group. Administration of PCA to normal mice resulted in a non-significant change in liver antioxidant status in comparison with the normal control group.

\section{TERT gene expression}

The relative gene expression of TERT in liver tissues of the different groups was shown in Fig. 3. Profound upregulation was recorded in TERT gene expression in the DMBA group in comparison to the normal control group. In contrast, administration of PCA to DMBA group effectively $(p<0.001)$ decreased TERT gene expression, by $-75 \%$, as compared to carcinogen group.

\section{Discussion}

Our results demonstrated that the PCA possesses antioxidant properties and it could serve as free radical inhibitors, scavenger or acting possibly as primary antioxidants. In addition, the PCA also protects liver carcinogenesis by inhibiting the antioxidant cascade and TERT expression in a mouse model administrated DMBA.

In the current study, a detectable decrease in body weight and an elevation in relative liver weight were noticed in DMBA group in comparison to normal group. This phenomena is well correlated with the proliferation of the hepatic cells upon DMBA administration and this agreed with an earlier study ${ }^{27)}$. The PCA treatment markedly increased body weight and decreased the mean relative liver weight in comparison to the DMBA group and these results may contribute more to effective antitumor action. Also, the current study recorded an elevation in the activities of hepatic marker enzymes; ALT, AST, $\gamma \mathrm{GT}$ and $\mathrm{T}$. bilirubin level with a decrease in albumin level in the DMBA group with respect to that of control group. These results are reflecting impairment in the liver function which results in an increase in these enzymes leakage into the bloodstream ${ }^{28)}$. These results are consistence with those obtained previously ${ }^{29)}$. The increase in the activity of $\gamma \mathrm{GT}$ may be attributed to the progression of carcinogenesis, since its activity is correlated with the rate of tumor growth and differentiation ${ }^{30)}$.

However, the PCA-treatment significantly reversed the hepatic marker enzymes activities to their near-normal levels and this treatment finally attenuated DMBAinduced liver carcinogenesis. PCA may aid in liver cell regeneration, preserving membrane integrity, thereby suppressing the leakage of the enzymes ${ }^{31}$. Moreover, the PCA was reported to decrease the liver marker en- zymes in lipopolysaccharide-induced rat hepatic damage via its anti-lipid peroxidative activity ${ }^{32)}$. AFP is a tumor marker which can be developed by regenerating hepatic tumors $^{33)}$. DMBA group displayed a marked increase in AFP level that can be related to the presence of premalignant liver modifications or genetic alterations that resulted from DMBA administration. The decreased level of AFP in DMBA-induced mice treated with PCA may reflect its anticancer potential in comparison with DMBA group.

The antioxidant enzymes are the first-line defense against oxidative damage and therefore offer protection against the harmful outcomes ${ }^{34)}$. In the current study, DMBA administration caused a marked elevation in MDA level due to excessive formation of free radicals. At the same time, there was a depletion in intracellular GSH content, associated with a decrease in the antioxidant enzymes activity (SOD and CAT). Similar results have been reported $^{35)}$. Meanwhile, the PCA treatment after DMBA administration significantly decreased the MDA level and increased the activities of SOD and CAT, and cellular GSH content as compared to DMBA group and these may be attributed to the powerful antioxidant activity and intrinsic free radical scavenging effects of PCA that inhibit the metabolic activated carcinogens or assist the excretion of carcinogenic metabolites of $\mathrm{DMBA}^{36)}$

Our results indicated that, the PCA can effectively inhibit $\mathrm{DPPH}^{\bullet}$ and $\mathrm{ABTS}^{+\bullet}$ in vitro that reflect its antioxidant effect. PCA may exert its antioxidant activity by scavenging free radicals through donation of hydrogen atom $\left(\mathrm{H}^{*}\right)$ or electron $(\mathrm{e})^{37)}$.

The results of RT-PCR of TERT mRNA indicated that the TERT expression was up-regulated in DMBA administrated group. This TERT up-regulation is a biological feature of preneoplastic lesions that evolve to hepatocellular carcinoma (HCC) in mice liver ${ }^{38)}$. TERT expression induces the growth of tumor by attuning the growth controlling genes expression and accelerating proliferation of the cell ${ }^{39)}$.

The TERT gene expression was decreased in response to PCA treatment without significantly affecting the expression of GAPDH, suggesting that the suppression of TERT expression by PCA is partially responsible for its anticancer activity. Approximately $90 \%$ of human cancers exhibit telomerase activity reactivation ${ }^{40)}$, therefore, telomerase is an attractive target for developing novel anticancer therapeutics selectively targeting the telomerase.

In conclusion, we showed that the PCA possesses antioxidant properties. In addition, the PCA also protects hepatocytes from carcinogenesis by inhibiting the antioxidant cascade and TERT expression in a mouse model administrated DMBA. It might be possible for PCA to use as a natural antioxidant and antitumor agent. 
Conflict of Interest:

The authors declare no conflict of interests.

\section{References}

1) Kozlov, M. V., Urnysheva, V. V., and Shishkina, L. N. (2008) Interconnection of parameters of regulation system of lipid peroxidation and of morphophysiological parameters of mouse liver. $\mathrm{Zh}$ Evol. Biokhim. Fiziol. 44: 398-402.

2) Grandhi, M. S., Kim, A. K., Ronnekleiv-Kelly, S. M., Kamel, I. R. Ghasebeh, M. A., and Pawlik, T. M. (2016) Hepatocellular carcinoma: From diagnosis to treatment. Surg. Oncol. 25: 74-85.

3) Smithgall, T. E., Harvey, R. G., and Penning, T. M. (1988) Oxidation of the trans-3,4-dihydrodiol metabolites of the potent carcinogen 7,12-dimethylbenz(a)anthracene and other benz(a)anthracene derivatives by 3a-hydroxysteroid-dihydrodiol dehydrogenase: Effects of methyl substitution on velocity and stereochemica. Cancer Res. 48: 1227-1232.

4) Digiovanni, J., and Juchau, M. R. (1980) Biotransformation and Bioactivation of 7,12-Dimethylbenz[a]anthracene (7,12-DMBA). Drug Metab. Rev. 11: 61-101.

5) Muqbil, I., and Banu, N. (2006) Enhancement of pro-oxidant effect of 7,12-dimethylbenz (a) anthracene (DMBA) in rats by preexposure to restraint stress. Cancer Lett. 240: 213-220.

6) Choi, E. J. (2008) Antioxidative effects of hesperetin against 7,12-dimethylbenz(a)anthracene-induced oxidative stress in mice. Life Sci. 82: 1059-1064.

7) Girolami, F., Abbadessa, G., Racca, S., Spaccamiglio, A., Piccione, F., Dacasto, M., Carletti, M., Gardini, G., Di Carlo, F., and Nebbia, C. (2008) Time-dependent acetylsalicylic acid effects on liver CYP1A and antioxidant enzymes in a rat model of 7,12-dimethylbenzanthracene (DMBA)-induced mammary carcinogenesis. Toxicol. Lett. 181: 87-92.

8) Shukla, S., and Mehta, A. (2015) Anticancer potential of medicinal plants and their phytochemicals: a review. Rev. Bras. Bot. 38: 199-210.

9) Beevi, S. S., Mangamoori, L. N., and Gowda, B. B. (2012) Polyphenolics profile and antioxidant properties of Raphanus sativus L. Nat. Prod. Res. 26: 557-563.

10) Kwak, J. H., Kim, H. J., Lee, K. H., Kang, S. C., and Zee, O. P. (2009) Antioxidative iridoid glycosides and phenolic compounds from Veronica peregrina. Arch. Pharm. Res. 32: 207-213.

11) Tanaka, T., Kojima, T., Kawamori, T., Yoshimi, N., and Mori, H. (1993) Chemoprevention of Diethylnitrosamine-induced Hepatocarcinogenesis by a Simple Phenolic Acid Protocatechuic Acid in Rats. Cancer Res. 53: 2775-2779.

12) Kim, N. W., Piatyszek, M. A., Prowse, K. R., Harley, C. B., West, M. D., Ho, P. L. C., Coviello, G. M., Wright, W. E., Weinrich, S. L., and Shay, J. W. (1994) Specific association of human telomerase activity with immortal cells and cancer. Science 266: 2011-2015.

13) Marian, C. O., Wright, W. E., and Shay, J. W. (2010) The effects of telomerase inhibition on prostate tumor-initiating cells. Int. J. Cancer 127: 321-331.

14) Blackburn, E. H. (2000) Telomere states and cell fates. Nature 408 53-56.

15) Meyerson, M., Counter, C. M., Eaton, E. N., Ellisen, L. W., Steiner, P., Caddle, S. D., Ziaugra, L., Beijersbergen, R. L., Davidoff, M. J., Qingyun, L., Bacchetti, S., Haber, D. A., and Weinberg, R. A. (1997) hEST2, the putative human telomerase catalytic subunit gene, is up- regulated in tumor cells and during immortalization. Cell 90 785-795.

16) Mansour, A., Daba, A., Baddour, N., El-Saadani, M., and Aleem, E. (2012) Schizophyllan inhibits the development of mammary and hepatic carcinomas induced by 7,12 dimethylbenz $(\alpha)$ anthracene and decreases cell proliferation: Comparison with tamoxifen. J. Cancer Res. Clin. Oncol. 138: 1579-1596.

17) OCDE. (2008). Oecd/Ocde, OECD Guidelined for The Testing of Chemicals. Acute oral toxicity. Up and Down Procedure (UDP). https://ntp.niehs.nih.gov/iccvam/suppdocs/feddocs/oecd/oecdtg425. pdf

18) Liu, C. L., Wang, J. M., Chu, C. Y., Cheng, M. T., and Tseng, T. H (2002) In vivo protective effect of protocatechuic acid on tert-butyl hydroperoxide-induced rat hepatotoxicity. Food Chem. Toxicol. 40: 635-641.

19) Beutler, E., Duron, O., and Kelly, B. M. (1963) Improved method for the determination of blood glutathione. J. Lab. Clin. Med. 61: 882-888.

20) Nishikimi, M., Appaji Rao, N., and Yagi, K. (1972) The occurrence of superoxide anion in the reaction of reduced phenazine methosulfate and molecular oxygen. Biochem. Biophys. Res. Commun. 46: 849-854.

21) Aebi, H. (1984) Catalase in vitro. Methods Enzymol. 105: 121-126.

22) Fossati, P., Prencipe, L., and Berti, G. (1980) Use of 3,5-dichloro2-hydroxybenzenesulfonic acid/4-aminophenazone chromogenic system in direct enzymic assay of uric acid in serum and urine. Clin. Chem. 26: 227-231.

23) Draper, H. H., and Hadley, M. (1990) Malondialdehyde determination as index of lipid Peroxidation. Methods Enzymol. 186: 421-431.

24) Re, R., Pellegrini, N., Proteggente, A., Pannala, A., Yang, M., and Rice-Evans, C. (1999) Antioxidant activity applying an improved ABTS radical cation decolorization assay. Free Radic. Biol. Med. 26: 1231-1237.

25) Mavi, A., Terzi, Z., Ozgen, U., Yildirim, A., and Coşkun, M. (2004) Antioxidant properties of some medicinal plants: Prangos ferulacea (Apiaceae), Sedum sempervivoides (Crassulaceae), Malva neglecta (Malvaceae), Cruciata taurica (Rubiaceae), Rosa pimpinellifolia (Rosaceae), Galium verum subsp. verum (Rubiaceae), Urtica di. Biol. Pharm. Bull. 27: 702-5.

26) Livak, K. J., and Schmittgen, T. D. (2001) Analysis of relative gene expression data using real-time quantitative PCR and the 2-Delta Delta-CT method. Methods 25: 402-408.

27) Bedi, P. S., and Priyanka, S. (2012) Effects of garlic against 7-12, Dimethyl benzanthracene induced toxicity in Wistar albino rats. Asian J. Pharm. Clin. Res. 5: 170-173.

28) Arora, R., Bhushan, S., Kumar, R., Mannan, R., Kaur, P., Singh, A P., Singh, B., Vig, A. P., Sharma, D., and Arora, S. (2014) Hepatic dysfunction induced by 7,12- Dimethylbenz $(\alpha)$ anthracene and its obviation with erucin using enzymatic and histological changes as indicators. PLoS One 9: 1-12.

29) El Kholy, W., Serag, H., and Zakaria, A. (2013) The Potency of some Natural Products on Dimethyl Benz ( A ) antheracene ( DMBA ) Induced Hepatotoxicity in Rats. Egypt. J. Hosp. Med. 53: 1036-1048.

30) Dakrory, A. I., Fahmy, S. R., Soliman, A. M., Mohamed, A. S., and Amer, S. A. M. (2015) Protective and curative effects of the sea cucumber Holothuria atra extract against DMBA-induced Hepatorenal diseases in rats. Biomed Res. Int. 2015:563652, 11 pages.

31) Chandra Mohan, K. V. P., Kumaraguruparan, R., Prathiba, D., and Nagini, S. (2006) Modulation of xenobiotic-metabolizing enzymes and redox status during chemoprevention of hamster buccal carcinogenesis by bovine lactoferrin. Nutrition 22: 940-946.

32) Lin, w., L., Hsieh, Y. J., Wang, C. J., Chou, F., P., Tseng, T.-H., and Cheng, M. T. (2003) Hibiscus protocatechuic acid inhibits lipopolysaccharide-induced rat hepatic damage. Arch. Toxicol. 77: 42-47.

33) Panova, I. G., Bezzubenko, Y. V., Tatikolov, A. S., Poltavtseva, R. A., Ivanets, T. Y., and Sukhikh, G. T. (2018) Alpha-Fetoprotein in Retina and Lens of the Human Eye at Early Stages of Prenatal Development. J. Evol. Biochem. Physiol. 54: 119-122.

34) Krishnamurthy, P., and Wadhwani, A. (2012) Antioxidant Enzymes and Human Health. In Antioxidant Enzyme 3:1-17.

35) Koul, A., Arora, N., and Tanwar, L. (2010) Lycopene mediated modulation of 7,12 dimethlybenz (A) anthracene induced hepatic clastogenicity in male Balb/c mice. Nutr. Hosp. 25: 304-310.

36) Wang, D., Zhao, Y., Jiao, Y., Yu, L., Yang, S., and Yang, X. (2012) Antioxidative and hepatoprotective effects of the polysaccharides from Zizyphus jujube cv. Shaanbeitanzao. Carbohydr. Polym. 88: 1453-1459. 
37) Li, X., Wang, X., Chen, D. and Chen, S. (2011) Antioxidant Activity and Mechanism of Protocatechuic Acid in vitro. Funct. Foods Heal. Dis. 1: 232-244.

38) Chen, Y., and Kong, Q. (2010) Nuclear translocation of telomerase reverse transcriptase: A critical process in chemical induced hepatocellular carcinogenesis. Neoplasma 57: 222-227.
39) Khattar, E., Kumar, P., Liu, C. Y., Can Akincilar, S., Raju, A., Lakshmanan, M., Maury, J. J. P., Qiang, Y., Li, S., Tan, E. Y., Hui, K. M., Shi, M., Loh, Y. H., and Tergaonkar, V. (2016) Telomerase reverse transcriptase promotes cancer cell proliferation by augmenting tRNA expression. J. Clin. Invest. 126: 4045-4060.

40) Janknecht, R. (2004) On the road to immortality: hTERT upregulation in cancer cells. FEBS Lett. 564: 9-13. 